\title{
Richter's Syndrome with Hypercalcemia Induced by Tumor-Associated Production of Parathyroid Hormone-Related Peptide
}

\author{
Naoki Watanabe ${ }^{a}$ Hajime Yasuda $^{a}$ Soji Morishita ${ }^{b}$ Yasuo Aota ${ }^{a, c}$ \\ Junichi Tomomatsu ${ }^{d}$ Masaru Tanaka $^{a}$ Akimichi Ohsaka $^{b}$ \\ Norio Komatsu ${ }^{a}$ \\ ${ }^{a}$ Division of Hematology, Department of Medicine, Juntendo University School of

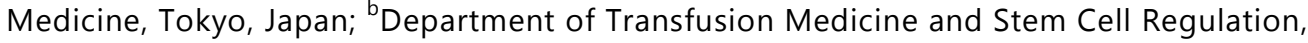 \\ Juntendo University School of Medicine, Tokyo, Japan; 'Department of Internal Medicine, \\ Kohsei Chuo General Hospital, Tokyo, Japan; ${ }^{d}$ Department of Medical Oncology, The \\ Cancer Institute Hospital of Japanese Foundation for Cancer Research, Tokyo, Japan
}

\section{Keywords}

Small lymphocytic leukemia · Chronic lymphocytic leukemia · Hypercalcemia · Parathyroid hormone-related peptide $\cdot$ Richter's syndrome

\begin{abstract}
Humoral hypercalcemia due to parathyroid hormone-related peptide (PTHrP) elevation is a well-known complication of various malignancies, but the situation is rare concerning hematological malignancies except for adult T-cell leukemia/lymphoma. We report a case of Richter's syndrome with humoral hypercalcemia, and demonstrate by reverse transcription polymerase chain reaction (RT-PCR) that peripheral blood PTHrP levels were 2,500-fold higher compared to healthy controls. PTHrP production by tumor cells in chronic lymphocytic leukemia (CLL) and Richter's syndrome has been previously demonstrated by nonquantitative methods such as immunohistochemistry and northern blot analysis, but this is the first report using the RT-PCR method. The presented case did not have hypercalcemia when initially
\end{abstract}


Watanabe et al.: Richter's Syndrome with Hypercalcemia Induced by Tumor-Associated Production of Parathyroid Hormone-Related Peptide

diagnosed as small lymphocytic lymphoma (SLL), and as reported earlier, the development of hypercalcemia may be an indication of the transformation to Richter's syndrome in patients with CLL/SLL.

\section{Introduction}

Hypercalcemia is a well-known complication of various malignancies. Humoral hypercalcemia of malignancy (HHM) is one of the mechanisms of hypercalcemia in malignancy, in which calcium liberation from the skeleton occurs due to a humoral mediator. The most frequently found mediator of HHM is parathyroid hormone-related peptide (PTHrP) [1]. HHM due to elevation of PTHrP is rare in hematological malignancies, except for adult T-cell leukemia/lymphoma (ATLL) [2]. Until now, very few reports of HHM occurring in chronic lymphocytic leukemia (CLL)/small lymphocytic leukemia (SLL) and Richter's syndrome exist. Herein, we report a rare case of Richter's syndrome with hypercalcemia induced by tumor-associated production of PTHrP.

\section{Case Presentation}

A 59-year-old male was referred to our hospital due to intra-abdominal lymphadenopathy in August 2010. He presented with a white blood cell (WBC) count of $5.6 \times 10^{9} / \mathrm{L}$, of which $76 \%$ consisted of mature lymphocytes. These cells were lambda light chain restricted and positive for CD5, CD19, CD23, and weakly positive for CD20. Peripheral blood IgH/BCL-1 analysis was negative by fluorescence in situ hybridization (FISH). Systemic lymphadenopathy and splenomegaly were detected by computed tomography (CT). A right cervical lymph node biopsy showed diffuse infiltration of small-to-medium-sized mature-appearing lymphocytes, and the surface antigen expression pattern was the same as that of the lymphocytes of the peripheral blood. Southern blot analysis revealed IGH-JH gene rearrangement. The bone marrow was hypercellular, with $90 \%$ accounting for predominantly mature lymphocytes. Lymph node chromosome analysis was not possibly due to an unobtainable metaphase, and FISH analysis showed no deletion of 17p13.1. Serum calcium level was $9.8 \mathrm{mg} / \mathrm{dL}$ (normal range, 8.8-10.6). Thus, a diagnosis of SLL was established. In July 2011, WBC count rose to $17.2 \times 10^{9} / \mathrm{L}$ with $87.5 \%$ consisting of mature lymphocytes, hemoglobin decreased to $9.8 \mathrm{~g} / \mathrm{dL}$, and platelet count decreased to $68 \times 10^{9} / \mathrm{L}$. The patient received 5 courses of FC (fludarabine, cyclophosphamide) therapy, but only a transient response was observed, and he subsequently received 6 courses of RB (rituximab, bendamustine) therapy, which led to a partial remission. However, disease progression was again observed in February 2013, and he presented with anorexia and abdominal bloating, WBC count rose to $18.3 \times 10^{9} / \mathrm{L}$ with $80 \%$ consisting of mature lymphocytes, and calcium levels were elevated at $13.2 \mathrm{mg} / \mathrm{dL}$. Whole-body CT scans revealed no osteolytic lesions. Serum 25-hydroxyvitamin D was 11 $\mathrm{ng} / \mathrm{mL}$ (normal range, 7-41). Serum intact PTH level was $8 \mathrm{pg} / \mathrm{mL}$ (normal range, 10-65 $\mathrm{pg} / \mathrm{mL}$ ) and decreased, whereas serum PTHrP was increased at $9.3 \mathrm{pmol} / \mathrm{L}$ (normal range, $<1.0$ ). At this point, we examined PTHrP messenger ribonucleic acid (mRNA) expression levels by reverse transcription polymerase chain reaction (RT-PCR) in the peripheral blood of our patient and 3 healthy subjects for comparison. Total RNA was extracted from the peripheral blood using QIAamp RNA Blood Mini Kit (Qiagen) according to the manufacturer's instructions. RNA was analyzed by qPCR performed with THUNDERBIRD SYBR qPCR Mix 


\section{Case Reports in Oncology}

Watanabe et al.: Richter's Syndrome with Hypercalcemia Induced by Tumor-Associated Production of Parathyroid Hormone-Related Peptide

(Toyobo) in a CFX96 Real-Time system (BioRad). Quantification was performed by the relative standard curve method against the control GAPDH. The patient's expression levels of PTHrP were found to be approximately 2,500-fold higher than that of healthy subjects. There were no differences between the expression levels of PTHrP among the healthy subjects. Thus, HHM induced by tumor-associated production of PTHrP was demonstrated. At the time of the development of hypercalcemia, progressive para-aortic lymphadenopathy was detected by CT, and a CT-guided para-aortic lymph node needle biopsy was carried out revealing a large-cell lymphoma with the same surface antigen expression pattern as when he was initially diagnosed as SLL, and thus transformation to Richter's syndrome was diagnosed. FISH analysis showed no deletion of $17 \mathrm{p} 13.1$. He received R-CHOP (rituximab, cyclophosphamide, doxorubicin, vincristine, and prednisone) therapy to which he was refractory, and he died of disease progression in April 2013.

\section{Discussion}

Hypercalcemia is a well-known complication of many malignancies. Some of the known mechanisms of hypercalcemia of malignancy are direct bone infiltration of tumor cells, elevation of humoral mediators such as PTHrP and 1,25-dihydroxyvitamin D, and ectopic secretion of PTH by tumor cells. In solid cancers, the incidence of hypercalcemia is most common in breast and renal carcinomas, followed by squamous cell carcinomas of any origin [1]. In hematological malignancies, the incidence of hypercalcemia is prevalent in ATLL and multiple myeloma [3], but only few reports of hypercalcemia in patients with CLL/SLL or Richter's syndrome exist [4]. Hypercalcemia in ATLL usually occurs due to elevation of PTHrP [2]. In ATLL, the HTLV-1 genome encodes a protein that activates the PTHrP promoter so that the generation of PTHrP encoding mRNA is increased and leads to a high production of PTHrP by the tumor cells [5, 6]. Hypercalcemia in CLL/SLL and Richter's syndrome has also been reported to be attributed to an elevated PTHrP, but unlike ATLL, the underlying mechanisms remain unclear. Among the few reports of CLL/SLL and Richter's syndrome developing HHM, reports actually demonstrating that PTHrP was produced from the tumor cells are even fewer. In 1 CLL case report, PTHrP production by tumor cells was demonstrated by Northern blot analysis of PTHrP mRNA in the liver, lymph nodes, spleen, and bone [7]. In another report of CLL, the expression of PTHrP by the leukemic cells was demonstrated by means of immunohistochemistry [8]. For the first time, we analyzed PTHrP levels in Richter's syndrome by the RT-PCR method. In comparison with Northern blot analysis and immunohistochemistry, RT-PCR is extremely quantitative, and we found that the patient's expression levels of PTHrP were approximately 2,500-fold higher in the peripheral blood compared to controls.

Another valuable aspect of this report is that we were able to observe calcium levels throughout the entire clinical course of this case. The patient's serum calcium levels increased only after transformation to Richter's syndrome. Thus, Hypercalcemia in patients with CLL may indicate transformation to Richter's syndrome as previously reported $[9,10]$. Richter's syndrome occurs in about $5 \%$ of the patients with CLL, and transformation to Richter's syndrome has been associated with a dismal prognosis, with a median survival of 5-8 months [11].

In conclusion, we report a case of Richter's syndrome with HHM induced by PTHrP. For the first time, we demonstrate by the RT-PCR method that PTHrP was 2,500-fold higher compared to healthy controls, and that PTHrP was most probably produced from the trans- 
Watanabe et al.: Richter's Syndrome with Hypercalcemia Induced by Tumor-Associated Production of Parathyroid Hormone-Related Peptide

formed cells of Richter's syndrome. PTHrP elevation and hypercalcemia in patients with CLL/SLL may indicate Richter's syndrome, and in such cases, serum intact PTH and PTHrP should be examined along with a pathological reevaluation.

\section{Statement of Ethics}

This study was approved by the institutional review board of Juntendo University School of Medicine. Written informed consent was obtained from the patient and healthy subjects prior to sample collection.

\section{Disclosure Statement}

The authors state that they have no conflicts of interest to disclose.

\section{References}

1 Sternlicht H, Glezerman IG: Hypercalcemia of malignancy and new treatment options. Ther Clin Risk Manag 2015;11:1779-1788.

2 Seymour JF, Gagel RF: Calcitriol: the major humoral mediator of hypercalcemia in Hodgkin's disease and non-Hodgkin's lymphomas. Blood 1993;82:1383-1394.

-3 Firkin F, Seymour JF, Watson AM, Grill V, Martin TJ: Parathyroid hormone-related protein in hypercalcaemia associated with haematological malignancy. Br J Haematol 1996;94:486-492.

4 Vaturi M, Prokocimer M, Sidi Y: Hypercalcemia in chronic lymphatic leukemia patients. Am J Hematol 1996;53:245-247.

5 Fukumoto S, Matsumoto T, Ikeda K, Yamashita T, Watanabe T, Yamaguchi K, Kiyokawa T, Shibuya N, Ogata E: Clinical evaluation of calcium metabolism in adult T-cell leukemia/lymphoma. Arch Intern Med 1988;148:921-925.

6 Watanabe T, Yamaguchi K, Takatsuki K, Osame M, Yoshida M: Constitutive expression of parathyroid hormone-related protein gene in human T cell leukemia virus type 1 (HTLV-1) carriers and adult T cell leukemia patients that can be trans-activated by HTLV-1 tax gene. J Exp Med 1990;172:759-765.

-7 Fain 0, el M'Selmi A, Dosquet C, Meseure D, Lejeune F, Garel JM, Thomas M: Hypercalcaemia in B cell chronic lymphocytic leukaemia. Br J Haematol 1994;87:856-858.

$>8$ Seymour JF, Keating MJ: Parathyroid hormone related protein in hypercalcaemia in CLL. Br J Haematol 1995;89:685-686.

\9 Beaudreuil J, Lortholary O, Martin A, Feuillard J, Guillevin L, Lortholary P, Raphael M, Casassus P: Hypercalcemia may indicate Richter's syndrome: report of four cases and review. Cancer 1997;79:1211-1215.

10 Freeman NJ, Holik D: Uncommon syndromes and treatment manifestations of malignancy: Case 3. Richter's syndrome heralded by refractory hypercalcemia. J Clin Oncol 2003;21:170-172.

$\checkmark 11$ Tsimberidou AM, Keating MJ: Richter syndrome: biology, incidence, and therapeutic strategies. Cancer 2005;103:216-228. 